\title{
Factores de riesgo asociados a estancia hospitalaria prolongada en pacientes con falla cardiaca aguda
}

\author{
Risk factors associated with prolonged hospital stay in patients with acute heart failure \\ Lina Arbeláez-Collazos, Lauren Calvo-Betancourt, Margarita Valencia-Mejía, Marietta Juan, \\ Nicolás Amaya, Emilio Juan, Alejandro Mariño y Ángel García-Peña* \\ Departamento de Medicina Interna, Hospital Universitario San Ignacio-Pontificia Universidad Javeriana, Bogotá, Colombia
}

\begin{abstract}
Resumen
Introducción: La falla cardiaca aguda es un motivo frecuente de consulta a urgencias, genera estancias hospitalarias prolongadas y altos costos para el sistema de salud. Objetivo: Determinar los factores asociados a estancia hospitalaria prolongada en pacientes hospitalizados por falla cardiaca aguda en un hospital universitario. Métodos: Estudio de cohorte retrospectivo, se incluyeron pacientes adultos con falla cardiaca aguda. Se obtuvieron variables demográficas, comorbilidades y resultados de laboratorios de rutina. Se definió hospitalización prolongada, como una estancia mayor a 5 y 10 días respectivamente. Resultados: Un total de 776 pacientes fueron incluidos en el análisis, 56\% eran hombres, el promedio de edad fue de 71.5 años, fracción de eyección de 39.8\%. Los factores asociados con estancia hospitalaria prolongada fueron: edad, elevación de troponina, hiperglucemia y albúmina < $3 \mathrm{~g} / \mathrm{dl}$. Para el corte de 10 días adicionalmente se identificaron: presión arterial sistólica, frecuencia cardiaca y elevación de péptidos natriuréticos. Conclusiones: La evaluación integral de variables clínicas y resultados de laboratorio es útil para identificar pacientes con mayor riesgo de estancias hospitalarias prolongadas.
\end{abstract}

Palabras clave: Falla cardiaca. Estancia hospitalaria. Riesgo.

\begin{abstract}
Introduction: Acute heart failure is a frequent reason for consulting to emergency department, it generates long hospital stays and high costs for the health system. Objective: To determine the associated factors with prolonged hospital stay in patients hospitalized for acute heart failure in a teaching hospital. Methods: Retrospective cohort study, adult patients with acute heart failure were included. Demographic variables, comorbidities, and routine laboratory results were obtained. Prolonged hospitalization was defined as a stay greater than 5 and 10 days, respectively. Results: $A$ total of 776 patients were included in the analysis, 56\% were men, the mean age was 71.5 years, and ejection fraction was $39.8 \%$. Factors associated with prolonged hospital stay were: age, elevated troponin, hyperglycemia, and albumin < $3 \mathrm{~g} / \mathrm{dl}$. For the 10-day cut-off, additionally, systolic blood pressure, heart rate and elevation of natriuretic peptides were identified. Conclusions: Comprehensive evaluation of clinical variables and laboratory results is useful to identify patients at increased risk for prolonged hospital stays.
\end{abstract}

Key words: Heart failure. Hospital stay. Risk.

\section{Correspondencia:}

*Ángel García-Peña

E-mail: angel.garcia@javeriana.edu.co;

aagarcia@ husi.org.co

Fecha de recepción: 26-03-2019

Fecha de aceptación: 31-07-2020

DOI: 10.24875/RCCAR.M21000022
Disponible en internet: 10-06-2021

Rev Colomb Cardiol. 2021;28(2):113-118

www.rccardiologia.com

0120-5633 / @ 2020 Sociedad Colombiana de Cardiología y Cirugía Cardiovascular. Publicado por Permanyer. Este es un artículo open access bajo la licencia CC BY-NC-ND (http://creativecommons.org/licenses/by-nc-nd/4.0/). 


\section{Introducción}

La falla cardiaca aguda es uno de los motivos más frecuentes de consulta al servicio de urgencias y la principal causa de hospitalización en mayores de 65 años ${ }^{1}$, grupo en el cual se reporta una incidencia aproximadamente de 10 casos por 10.000 personas ${ }^{1}$.

En Europa, la prevalencia de falla cardiaca aguda en población adulta oscila entre el $0.4 \%$ y el $2 \%$, situación que aumenta con la edad y que para los mayores de 70 años llega a más del 10\%. En Colombia, según datos del Ministerio de Salud y Protección Social, la prevalencia en la población general es de aproximadamente el $2.3 \%$, las consultas al servicio de urgencias por falla cardiaca aguda han aumentado en un $50 \%$ y las hospitalizaciones por esta se han incrementado en un $30 \%^{2}$.

La descompensación aguda conlleva altos gastos para el sistema de salud, los cuales se derivan de la consulta al servicio de urgencias, las hospitalizaciones prolongadas, el tratamiento farmacológico, la rehabilitación cardiaca y la discapacidad asociada ${ }^{1}$.

En los últimos años han surgido opciones diferentes a la hospitalización convencional, como la hospitalización en casa, las unidades de corta estancia y las unidades de observación, en las que se da un manejo temprano y dirigido a pacientes que cursan con falla cardiaca aguda con posibilidad de rápida recuperación, con miras a disminuir el ingreso a una hospitalización convencional y los costos que esta acarrea ${ }^{3-5}$. El problema radica en identificar a los candidatos a estos programas y diferenciarlos de aquellos que, por la presencia de factores de riesgo clínicos o paraclínicos, requerirán manejo intrahospitalario convencional.

Es importante identificar los factores asociados con una mayor estancia hospitalaria, lo cual permitirá a las instituciones de atención en salud diseñar estrategias para distribuir apropiadamente el uso de los recursos o incluso diseñar vías de atención diferenciales para disminuir la morbimortalidad y el deterioro funcional ${ }^{1}$. Por esta razón, el objetivo de este estudio fue evaluar la relación que existe entre las características de los pacientes que ingresan al servicio de urgencias por un episodio de falla cardiaca aguda con posterior estancia hospitalaria prolongada para manejo de la enfermedad en un hospital de alta complejidad de Colombia.

\section{Método}

Se llevó a cabo un estudio observacional, analítico, de cohorte, retrospectivo, en un hospital de alta complejidad en Colombia.
Se utilizó el registro institucional de falla cardiaca aguda, con un total de 776 pacientes que contaban con toda la información disponible para el análisis. Se siguió la recomendación de Peduzzi ${ }^{6}$ para una relación de variables: eventos de 1:10, con el objetivo de obtener en el análisis de regresión logística un error tipo I del $0.05 \%$, un error tipo 2 del $0,2 \%$ y un poder del $80 \%$, con significancia estadística de $p<0.05$.

Se incluyeron adultos ( $\geq 18$ años) con diagnóstico de falla cardiaca aguda definido en las primeras 24 horas del ingreso a urgencias y confirmado al momento del ingreso por un médico especialista (en medicina interna, emergencias, cardiología, medicina familiar o geriatría), o que tuvieran una estancia hospitalaria $>24$ horas, y de quienes la información de la historia clínica estuviera disponible para el estudio. Se excluyeron aquellos con antecedente de trasplante de cualquier órgano o médula, o en lista de trasplante cardiaco o con dispositivo de asistencia ventricular, o remitidos a otra institución en las primeras 24 horas.

La variable desenlace fue el tiempo de estancia hospitalaria prolongado, considerándose dos puntos de corte a valorar: $>5$ y 10 días de hospitalización. Esta variable se recolectó de forma cuantitativa; sin embargo, para el análisis se convirtió en variable dicotómica (0-1). Las variables demográficas, la comorbilidad y los datos clínicos relevantes se presentan en la tabla 1.

Se realizó una evaluación de los datos, recuperando la totalidad de los datos faltantes a partir del análisis de texto plano de la historia clínica. Se procedió a hacer el análisis descriptivo: medidas de tendencia central para variables cuantitativas, y conteos y proporciones para variables ordinales o categóricas. Para el análisis de asociación-predicción de los factores analizados, las variables desenlace fueron la estancia prolongada (5 y 10 días), dicotomizando la variable desenlace. Se realizó la evaluación mediante modelos univariados, bivariados y multivariados, teniendo en cuenta el desenlace de interés (hospitalización prolongada, variable dicotómica). Posteriormente, se cumplieron los supuestos para realizar un modelo de regresión logística con el objetivo de integrar la información bajo metodología stepwise buscando el modelo más parsimonioso.

\section{Resultados}

Se incluyeron 776 pacientes ingresados por falla cardiaca aguda, de los que 434 (56\%) eran hombres. La 
Tabla 1. Descripción general

\begin{tabular}{|c|c|}
\hline Variable & Valor \\
\hline Edad (años) & 71.5 (DE: 14.3) \\
\hline Mujeres & $342(44 \%)$ \\
\hline Hombres & $434(56 \%)$ \\
\hline FEVI (\%) & 39.8 (DE: 16.4) \\
\hline Presión arterial sistólica $(\mathrm{mmHg})$ & 127.8 (DE: 40.9) \\
\hline Frecuencia respiratoria (r.p.m) & 20.3 (DE: 4.9) \\
\hline Frecuencia cardiaca (I.p.m.) & 82.8 (DE: 21.1) \\
\hline $\mathrm{SaO}_{2}(\%)$ & 91.5 (DE: 6.1 ) \\
\hline $\begin{array}{l}\text { Comorbilidad (\%) } \\
\text { Hipertensión arterial } \\
\text { Diabetes mellitus } \\
\text { Dislipidemia } \\
\text { Falla cardiaca previa } \\
\text { Fibrilación auricular } \\
\text { EPOC } \\
\text { Enfermedad renal crónica } \\
\text { Enfermedad arterial periférica } \\
\text { Cáncer }\end{array}$ & $\begin{array}{c}69 \\
24 \\
19 \\
63 \\
29 \\
30 \\
26 \\
4 \\
6\end{array}$ \\
\hline $\begin{array}{l}\text { Hallazgos }(\%) \\
\text { Electrocardiograma con signos de } \\
\text { isquemia } \\
\text { Radiografía de tórax con signos } \\
\text { congestivos }\end{array}$ & $\begin{array}{c}8 \\
49\end{array}$ \\
\hline $\begin{array}{l}\text { Paraclínicos } \\
\text { Péptido natriurético (pg/dl) } \\
\text { Hemoglobina }(\mathrm{g} / \mathrm{dl}) \\
\text { Creatinina }(\mathrm{mg} / \mathrm{dl}) \\
\text { BUN }(\mathrm{mg} / \mathrm{dl}) \\
\text { Sodio }(\mathrm{mEq} / \mathrm{l}) \\
\text { Potasio }(\mathrm{mEq} / \mathrm{L}) \\
\text { Glucemia }(\mathrm{mg} / \mathrm{dl})\end{array}$ & $\begin{array}{c}2368 \text { (DE: 3672) } \\
13.1 \text { (DE: } 4.1) \\
1.48 \text { (DE: } 1.26) \\
29.1 \text { (DE: } 16.4) \\
\text { 135.3 (DE: 14.2) } \\
4.4 \text { (DE: } 3.8) \\
75.3 \text { (DE: } 74.9 \text { ) }\end{array}$ \\
\hline $\begin{array}{l}\text { Estancia hospitalaria } \\
\text { Tiempo promedio (días) }\end{array}$ & 10 (DE: 9) \\
\hline
\end{tabular}

BUN: nitrógeno ureico en sangre; DE: desviación estándar; EPOC: enfermedad pulmonar obstructiva crónica; FEVI: fracción de eyección del ventrículo izquierdo; I.p.m.: latidos por minuto; r.p.m.: respiraciones por minuto; $\mathrm{SaO}_{2}$ : saturación de oxígeno.

edad media fue de 71.5 años (desviación estándar [DE]: 14.3). El promedio de la fracción de eyección fue del $39.8 \%$ (DE: 16.4). La mayoría tenían antecedente de episodio de falla cardiaca aguda $(63 \%)$ y la principal comorbilidad observada fue hipertensión arterial (69\%) y enfermedad pulmonar obstructiva crónica (30\%). La tabla 2 muestra las medidas de efecto tras un análisis de regresión logística de los factores que podrían explicar el tiempo de estancia hospitalaria prolongado $>5$ días, y en la tabla 3 para más de 10 días, que lograron significancia estadística y quedaron incluidas en los modelos de regresión.

\section{Discusión}

En este estudio se identificaron factores independientes relacionados con tiempos prolongados de estancia hospitalaria. El reconocimiento de estos al momento del ingreso al servicio de urgencias puede ser útil para optimizar el manejo de recursos por paciente y disminuir el número ingresos no indicados a unidades de corta estancia ${ }^{3}$.

La población estudiada guarda similitud, en cuanto a edad y características demográficas, con las de los grandes registros internacionales de pacientes con falla cardiaca, pero difiere en el sexo, pues en nuestro estudio prevalece la población masculina ${ }^{1}$. El tiempo promedio de estancia hospitalaria fue de 10 días, la cual se encuentra por encima de la registrada por Ciapponi et al. ${ }^{4}$ en uno de los estudios más grandes realizados en América Latina, en el que encontraron un tiempo promedio de 6.9 días, así como la identificada en otros registros internacionales de falla cardiaca: 4.3 días en el registro $\mathrm{ADHERE}^{7}$ y 5.7 días en el OPTIMIZE $\mathrm{HF}^{1,2,5}$. Sin embargo, se conoce que existe una alta variabilidad en el tiempo de hospitalización por un episodio de falla cardiaca aguda entre las diferentes instituciones ${ }^{1}$. Esto, además, puede ser explicado por la alta morbilidad de los pacientes que acuden a nuestra institución y la gravedad de la falla cardiaca evidenciada por el promedio de péptidos natriuréticos y troponina positiva.

En este estudio, los factores de riesgo asociados a estancia prolongada fueron la edad, la presión arterial sistólica, la frecuencia cardiaca, los péptidos natriuréticos elevados, la troponina positiva, la albúmina $<3 \mathrm{~g} /$ dl y la glucemia (Tabla 1). Es lógico pensar que los pacientes mayores tendrán una estancia prolongada por sus enfermedades asociadas; la troponina y los péptidos natriuréticos elevados como biomarcadores de disfunción miocárdica serán buenos predictores, y la gravedad de la falla se correlacionará con el impacto sistémico que se traduce en hipoalbuminemia y las anormalidades de la glucosa. No obstante, al revisar la literatura, se observa que los resultados son muy variables en cada estudio (Tabla 4) 9-17. De forma consistente, se demuestra un menor riesgo de estancia hospitalaria prolongada con mayores valores de presión arterial sistólica, al igual que lo observado en los registros descritos previamente.

Una de las ventajas de este estudio es que identifica factores predictores de estancia prolongada en variables de laboratorio que pueden ser fácilmente evaluadas en urgencias, y permite la identificación rápida de 
Tabla 2. Hallazgos de la regresión logística (hospitalización > 5 días)

\begin{tabular}{|l|c|c|c|c|c|c|}
\hline Hospitalización $>\mathbf{5}$ días & Razón de disparidad & Error estándar & $\mathbf{z}$ & $\mathbf{P}>\mathbf{z}$ & \multicolumn{2}{|c|}{ Intervalo de confianza del $95 \%$} \\
\hline Edad (años) & 0.9737319 & 0.0066053 & -3.92 & 0.000 & 0.9608713 & 0.9867645 \\
\hline FEVI (\%) & 0.9899152 & 0.0053779 & -1.87 & 0.062 & 0.9794306 & 1.000 .512 \\
\hline Troponina positiva & 2.257068 & 0.5648457 & 3.25 & 0.001 & 1.382 .058 & 3.686 .068 \\
\hline Albúmina $<3$ g/dl & 2.019591 & 0.4993175 & 2.84 & 0.004 & 1.243 .985 & 3.278 .777 \\
\hline Glucemia & 1.003797 & 0.0012725 & 2.99 & 0.003 & 1.001 .306 & 1.006 .294 \\
\hline _cons & 1.781716 & 9.167 .571 & 5.60 & 0.000 & 6.499 .272 & 4.884 .412 \\
\hline
\end{tabular}

FEVI: fracción de eyección del ventrículo izquierdo.

Número de observaciones: 776. $\operatorname{LR} \chi 2(5)=53.87$. Prob $>\chi^{2}=0.0000$. Log likelihood $=-420.08$. Pseudo $R^{2}=0.06$.

Tabla 3. Hallazgos de la regresión logística (hospitalización > 10 días)

\begin{tabular}{|l|c|c|c|c|c|c|}
\hline Hospitalización $>\mathbf{1 0}$ días & Raźn de disparidad & Error estándar & $\mathbf{z}$ & $\mathbf{P}>\mathbf{z}$ & Intervalo de confianza del 95\% \\
\hline Edad (años) & .90720016 & 0.0056434 & -4.89 & 0.000 & 0.9610034 & 0.9831257 \\
\hline Presión arterial sistólica (mmHg) & 0.9866549 & 0.0031191 & -4.25 & 0.000 & 0.9805605 & 0.9927872 \\
\hline Frecuencia cardiaca (I.p.m.) & 1.011 .347 & 0.0038682 & 2.95 & 0.003 & 1.003 .794 & 1.018 .957 \\
\hline Péptido natriurético $>$ 500 mg/dl & 1.708 .302 & 0.3641662 & 2.51 & 0.012 & 1.124 .891 & 2.594 .293 \\
\hline Troponina positiva & 1.508 .854 & 0.3111981 & 1.99 & 0.046 & 1.007 .137 & 2.260 .507 \\
\hline Albúmina $<3$ g/dl & 1.930 .955 & 0.3880863 & 3.27 & 0.001 & 1.302 .256 & 2.863 .176 \\
\hline Glucemia & 1.002 .906 & 0.0010718 & 2.72 & 0.007 & 1.000 .808 & 1.005 .009 \\
\hline cons & 3.438 .308 & 2.322 .167 & 1.83 & 0.067 & 0.9150813 & 1.291 .903 \\
\hline
\end{tabular}

I.p.m.: latidos por minuto.

Número de observaciones: 776. $\operatorname{LR} \chi 2(7)=92.07$. Prob $>\chi^{2}=0.0000$. Log likelihood $=-452.82$. Pseudo $\mathrm{R}^{2}=0.09$.

aquellos pacientes que podrían requerir una estancia hospitalaria prolongada, lo que quizás pueda favorecer rutas de atención o prioridades diferenciales para asignación de recursos o personal para su manejo.

Existen limitaciones a los hallazgos descritos. Una de ellas es inherente al tipo de diseño y su carácter retrospectivo, y al desarrollo del estudio en un solo centro de referencia y alta complejidad, por lo que los resultados no podrían ser extrapolados a otros niveles de atención. No se evaluaron variables de confusión relacionadas con la gravedad de la falla cardiaca previa a la hospitalización ni aspectos demográficos, como el día de la semana, el horario de ingreso y el servicio tratante, que podrían mostrar diferencias en el abordaje o los resultados de las intervenciones; sin embargo, la adherencia a las guías de falla cardiaca institucional es superior al $85 \%$ en todos los servicios tratantes.
En el futuro se requieren estudios, de preferencia multicéntricos, que incluyan instituciones de todos los niveles de atención con el fin de obtener un panorama claro y real de la población de pacientes con falla cardiaca aguda en Colombia, para lograr evaluar el impacto de otras variables, la adherencia al tratamiento y los aspectos sociales relevantes que puedan mejorar la capacidad de predicción o identificación de pacientes en alto riesgo de hospitalización prolongada.

\section{Conclusiones}

La estancia hospitalaria prolongada en pacientes con falla cardiaca aguda es un fenómeno complejo con múltiples factores asociados. En este estudio se identificaron cinco variables principales que se correlacionaban con tiempos mayores de estancia hospitalaria prolongada, las cuales fueron fácilmente evaluadas y 
Tabla 4. Resumen de los estudios disponibles sobre factores de riesgo para estancia hospitalaria prolongada en pacientes con falla cardiaca aguda

\begin{tabular}{|c|c|c|}
\hline Autores & Definición de estancia prolongada & Factores asociados \\
\hline Formiga et al. ${ }^{9}, 2007$ & $>4$ días & Sexo femenino, clase funcional NYHA avanzada \\
\hline Alnajashi et al. ${ }^{11}, 2016$ & $\geq 6$ días & $\begin{array}{l}\text { Edad, primera hospitalización por falla cardiaca, lesión renal } \\
\text { aguda y fracción de eyección }(<35 \%)\end{array}$ \\
\hline Martín-Sánchez et al. ${ }^{12}, 2014$ & $>72$ horas & $\begin{array}{l}\text { EPOC, saturación de oxígeno basal a la llegada a urgencias } \\
<90 \% \text {, ingresar el jueves } \\
\text { Crisis hipertensiva como desencadenante de falla cardiaca } \\
\text { como factor protector }\end{array}$ \\
\hline Gyalai-Korpos et al. ${ }^{13}, 2015$ & $\begin{array}{l}\text { Estancia por encima del percentil } 75 \\
\text { Pacientes }>65 \text { años }\end{array}$ & Exacerbación infecciosa de EPOC \\
\hline Wright et al. ${ }^{14}, 2003$ & $>6$ días & $\begin{array}{l}\text { Edema, cambio de peso durante la estancia, duración de } \\
\text { tratamiento con diurético intravenoso, lesión renal aguda, } \\
\text { problemas respiratorios asociados, problemas sociales }\end{array}$ \\
\hline Krumholz et al. ${ }^{15}, 1999$ & Por encima del promedio de estancia & $\begin{array}{l}\text { Lesión renal previa, edema, fibrilación auricular, hiponatremia, } \\
\text { sonda vesical al momento del ingreso, inicio de antiarrítmicos } \\
\text { o warfarina }\end{array}$ \\
\hline Martín-Sánchez et al. ${ }^{3}, 2016$ & $>7$ días & $\begin{array}{l}\text { EPOC, ser portador de un dispositivo, lesión renal aguda al } \\
\text { ingreso, hiponatremia, anemia } \\
\text { Hipertensión arterial y falta de adherencia al tratamiento } \\
\text { como factores protectores }\end{array}$ \\
\hline Armola y Topp ${ }^{16}, 2001$ & $\geq 6$ días & $\begin{array}{l}\text { Número de médicos involucrados en el caso, valores de } \\
\text { sodio, clasificación NYHA avanzada }\end{array}$ \\
\hline Cotter et al. ${ }^{17}, 2015$ & $\begin{array}{l}\text { Según los tertiles de la institución } \\
\text { estudiada }\end{array}$ & $\begin{array}{l}\text { Mayor grado de disnea, diabetes mellitus, cardiopatía } \\
\text { valvular, creatinina o troponina elevadas, hemoglobina o sodio } \\
\text { bajos } \\
\text { Hipertensión arterial como factor protector }\end{array}$ \\
\hline Davison et al. ${ }^{10}, 2016$ & $\begin{array}{l}\text { Según los tertiles de la institución } \\
\text { estudiada }\end{array}$ & $\begin{array}{l}\text { Diabetes mellitus, angina de pecho, mayor IMC, mayor } \\
\text { frecuencia cardiaca, ortopnea, presión venosa yugular, BUN y } \\
\text { ácido úrico }\end{array}$ \\
\hline
\end{tabular}

BUN: nitrógeno ureico en sangre; EPOC: enfermedad pulmonar obstructiva crónica; IMC: índice de masa corporal; NYHA: New York Heart Association.

podrían ser de utilidad para definir el escenario de cuidado o ruta de atención en este tipo de pacientes.

\section{Financiamiento}

Hospital Universitario San Ignacio, Bogotá,
Colombia.

\section{Conflicto de intereses}

Los autores declaran no tener ningún conflicto de intereses.

\section{Responsabilidades éticas}

Protección de personas y animales. Los autores declaran que para esta investigación no se han realizado experimentos en seres humanos ni en animales.
Confidencialidad de los datos. Los autores declaran que han seguido los protocolos de su centro de trabajo sobre la publicación de datos de pacientes.

Derecho a la privacidad y consentimiento informado. Los autores declaran que en este artículo no aparecen datos de pacientes.

\section{Bibliografía}

1. Rodríguez-Artalejo F, Banegas Banegas JR, Guallar-Castillón P. Epidemiología de la insuficiencia cardíaca. Rev Esp Cardiol. 2004;57:163-70. Disponible en: https://doi.org/10.1016/S0300-8932(04)77080-3.

2. Jaramillo C, Gómez E, Hernández E, Saldarriaga C, Flórez N, Buitrago R, et al. Consenso colombiano de diagnóstico y tratamiento de la insuficiencia cardiaca crónica. Bogotá: Inversiones Tecnográficas S.A.S.; 2014. p. 7-9.

3. Martín-Sánchez FJ, Carbajosa V, Llorens P, Herrero P, Jacob J, Miró O, et al. Tiempo de estancia prolongado en los pacientes ingresados por insuficiencia cardiaca aguda. Gac Sanit. 2016;30:191-200. Disponible en: https://doi.org/10.1016/j.gaceta.2016.01.003.

4. Ciapponi A, Alcaraz A, Calderón M, Matta M, Chaparro M, Soto N, et al. Carga de enfermedad de la insuficiencia cardiaca en América Latina: revisión sistemática y metaanálisis. Rev Esp Cardiol. 2016;69:1051-60. 
Rev Colomb Cardiol. 2021;28(2)

5. López Pardo P, Socorro García A, Baztán Cortés JJ. Influence of length of hospital stay on mortality after discharge in older patients with acute medical diseases. Gac Sanit. 2016;30:375-8. Disponible en: https://doi. org/10.1016/j.gaceta.2016.04.008.

6. Peduzzi P, Concato J, Kemper E, Holford TR, Feinstem AR. A simulation study of the number of events per variable in logistic regression analysis. J Clin Epidemiol. 1996;49:1373-9. Disponible en: https://doi. org/10.1016/S0895-4356(96)00236-3.

7. Fonarow GC. The Acute Decompensated Heart Failure National Registry (ADHERE): opportunities to improve care of patients hospitalized with acute decompensated heart failure. Rev Cardiovasc Med. 2003;4 (Suppl 7):S21-30. Disponible en: http://www.ncbi.nlm.nih.gov/pub$\mathrm{med} / 14668697$.

8. Abraham WT, Fonarow GC, Albert NM, Stough WG, Gheorghiade M Greenberg $\mathrm{BH}$, et al. Predictors of in-hospital mortality in patients hospitalized for heart failure. insights from the Organized Program to Initiate Lifesaving Treatment in Hospitalized Patients With Heart Failure (OPTIMIZE-HF). J Am Coll Cardiol. 2008;52:347-56. Disponible en: https://doi. org/10.1016/j.jacc.2008.04.028

9. Formiga F, Chivite D, Manito N, Mestre AR, Llopis F, Pujol R. Admission characteristics predicting longer length of stay among elderly patients hospitalized for decompensated heart failure. Eur J Int Med. 2008:19:198202. Disponible en: https://doi.org/10.1016/j.ejim.2007.09.007

10. Davison BA, Metra M, Senger S, Edwards C, Milo O, Bloomfield DM, et al. Patient journey after admission for acute heart failure: length of stay, 30-day readmission and 90-day mortality. Eur $\mathrm{J}$ Heart Fail. 2016;18:1041-50. Disponible en: https://doi.org/10.1002/ejhf.540.

11. Alnajashi MA, Almasouda MA, Aldaham SA, Acuna JM, Zevallos JC. Association of gender and length of stay among Puerto Ricans hospita- lized with decompensated heart failure. Medicine (Baltimore). 2016;95:e4255. Disponible en: https://doi.org/10.1097/ MD. 0000000000004255

12. Martín-Sánchez FJ, Carbajosa V Llorens $P$, Herrero $P$ Jacob J, Pérez-Dura $\mathrm{M}$, et al. Estancia prolongada en pacientes ingresados por insuficiencia cardiaca aguda en la Unidad de Corta Estancia (estudio EPICA-UCE): factores asociados. Med Clin. 2014;143:245-51. Disponible en: https://doi.org/10.1016/j.medcli.2013.06.028

13. Gyalai-Korpos I, Ancusa O, Dragomir T, Tomescu MC, Marincu I. Factors associated with prolonged hospitalization, readmission, and death in elderly heart failure patients in western Romania. Clin Interv Aging. 2015;10:561-8. Disponible en: https://doi.org/10.2147/CIA. S79569.

14. Wright SP, Verouhis D, Gamble G, Swedberg K, Sharpe N, Doughty RN Factors influencing the length of hospital stay of patients with heart failure. Eur J Heart Fail. 2003;5:201-9. Disponible en: https://doi. org/10.1016/S1388-9842(02)00201-5.

15. Krumholz HM, Chen YT, Bradford WD, Cerese J. Variations in and correlates of length of stay in academic hospitals among patients with heart failure resulting from systolic dysfunction. Am J Manag Care. 1999;5:715-23.

16. Armola RR, Topp R. Variables that discriminate length of stay and readmission within 30 days among heart failure patients. Lippincotts Case Manag. 2001;6:246-55. Disponible en: https://doi.org/10.1097 / 00129234200111000-00005

17. Cotter G, Davison BA, Milo O, Bourge RC, Cleland JGF, Jondeau G et al. Predictors and associations with outcomes of length of hospital stay in patients with acute heart failure: results from VERITAS. J Cardiac Fail. 2016;22:815-22. Disponible en: https://doi.org/10.1016/j.cardfail.2015.12.017. 